\title{
Moving Arrows and Four Model Checking Results
}

\author{
Carlos Areces $^{1,2}$, Raul Fervari ${ }^{1}$ and Guillaume Hoffmann ${ }^{1}$ \\ 1 FaMAF, Universidad Nacional de Córdoba, Argentina \\ \{careces, fervari, hoffmann\}@famaf.unc.edu.ar \\ 2 CONICET, Argentina
}

\begin{abstract}
We study dynamic modal operators that can change the model during the evaluation of a formula. In particular, we extend the basic modal language with modalities that are able to swap, delete or add pairs of related elements of the domain, while traversing an edge of the accessibility relation. We study these languages together with the sabotage modal logic, which can arbitrarily delete edges of the model. We define a suitable notion of bisimulation for the basic modal logic extended with each of the new dynamic operators and investigate their expressive power, showing that they are all uncomparable. We also show that the complexity of their model checking problems is PSpace-complete.
\end{abstract}

\section{Introduction}

Modal logics [2,4] are particularly well suited to describe graphs, and this is fortunate as many situations can be modeled using graphs: an algebra, a database, the execution flow of a program or, simply, the arbitrary relations between a set of elements. This explains why modal logics have been used in many, diverse fields. They offer a well balanced trade-off between expressivity and computational complexity (model checking the basic modal language $\mathcal{M L}$ is only polynomial, while its satisfiability problem is PSpace-complete). Moreover, the range of modal logics known today is extremely wide, so that it is usually possible to pick and choose the right modal logic for a particular application.

But if we want to describe and reason about dynamic aspects of a given situation, e.g., how the relations between a set of elements evolve through time or through the application of certain operations, the use of modal logics (or actually, any kind of logic with classical semantics) becomes less clear. We can always resort to modeling the whole space of possible evolutions of the system as a graph, but this soon becomes unwieldy. It would be more elegant to use truly dynamic modal logics with operators that can mimic the changes that structure will undergo. This is not a new idea, and a clear example of this kind of logics is the sabotage logic introduced by Johan van Benthem in [12].

Consider the following sabotage game. It is played on a graph with two players, Runner and Blocker. Runner can move on the graph from node to accessible node, starting from a designated point, and with the goal of reaching a given 
final point. He should move one edge at a time. Blocker, on the other hand, can delete one edge from the graph, every time it is his turn. Of course, Runner wins if he manages to move from the origin to the final point in the graph, while Blocker wins otherwise. van Benthem discusses in [12] how to transform the sabotage game into a modal logic. van Benthem's original idea has been studied in several other works $[6,10]$ where the sabotage operator is defined as:

$$
\mathcal{M}, w \models\langle g s\rangle \varphi \text { iff there is a pair }(u, v) \text { of } \mathcal{M} \text { such that } \mathcal{M}_{\{(u, v)\}}^{-}, w \models \varphi,
$$

where $\mathcal{M}_{\{(u, v)\}}^{-}$is identical to $\mathcal{M}$ except that the edge $(u, v)$ has been removed from the accessibility relation.

It is clear that the $\langle g s\rangle$ operator changes the model in which a formula is evaluated. As van Benthem puts it, $\langle g s\rangle$ is an "external" modality that takes evaluation to another model, obtained from the current one by deleting some transition. It has been proved that solving the sabotage game is PSpace-hard, while the model checking problem of the associated modal logic is PSpace-complete and the satisfiability problem is undecidable. The logic fails to have both the finite model property and the tree model property $[6,10]$.

In this article, we will investigate various model changing operators. The first one, $\langle s w\rangle$, has the ability to swap the direction of a traversed arrow. The $\langle s w\rangle$ operator is a $\diamond$ operator - to be true at a state $w$ it requires the existence of an accessible state $v$ where evaluation will continue - but it changes the accessibility relation during evaluation - the pair $(w, v)$ is deleted, and the pair $(v, w)$ added to the accessibility relation. A picture will help understand the dynamics of $\langle s w\rangle$. The formula $\langle s w\rangle \diamond T$ is true in a model with two related states:
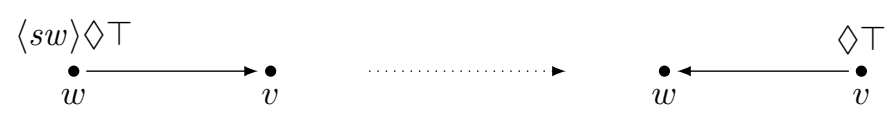

As we can see in the picture, evaluation starts at state $w$ with the arrow pointing from $w$ to $v$, but after evaluating the $\langle s w\rangle$ operator, it continues at state $v$ with the arrow now pointing from $v$ to $w$. We will investigate two other dynamic operators in this article. $\langle l s\rangle$, for local sabotage, is a $\diamond$ operator that destroys the traversed arrow, while $\langle b r\rangle$, for bridge, models the opposite situation: it adds an arrow to an inaccessible point of the model and moves over there.

We have chosen these model changing operators with the intention of covering a sufficiently varied sample of alternatives. The goal is to investigate whether the differences among them lead to different properties of the logics they defined, and how they vary in expressive power. Clearly, other operators could have been included in this exploration, and actually some alternative choices have been already investigated in the literature, e.g., the adjacent sabotage operator discussed in [10].

Summing up then, we will study and compare the expressive powers of $\mathcal{M L}(\langle s w\rangle), \mathcal{M L}(\langle g s\rangle), \mathcal{M L}(\langle l s\rangle)$ and $\mathcal{M L}(\langle b r\rangle)$, and we provide complexity results for their model checking problems. 


\section{Syntax and Semantics}

The syntax of the dynamic modal logics we will study is a straightforward extension of the basic modal logic (see [2]):

Definition 1 (Syntax). Let PROP be a countable, infinite set of propositional symbols. Then the set FORM of formulas over PROP is defined as:

$$
\text { FORM }::=\perp|p| \neg \varphi|\varphi \wedge \psi| \diamond \varphi,
$$

where $p \in \mathrm{PROP}, \diamond \in\{\diamond,\langle s w\rangle,\langle g s\rangle,\langle l s\rangle,\langle b r\rangle\}$ and $\varphi, \psi \in \mathrm{FORM}$. Other operators are defined as usual. In particular, $\boldsymbol{\square} \varphi$ is defined as $\neg \neg \varphi$.

Formulas of the basic modal language $\mathcal{M L}$ are those that contains only the $\diamond$ operator beside the Boolean operators. We call $\mathcal{M L}(\checkmark)$ to the extension of $\mathcal{M L}$ allowing also the operator, for $\in\{\langle s w\rangle,\langle g s\rangle,\langle l s\rangle,\langle b r\rangle\}$.

Semantically, formulas of $\mathcal{M L}(\langle s w\rangle), \mathcal{M L}(\langle g s\rangle), \mathcal{M L}(\langle l s\rangle)$ and $\mathcal{M L}(\langle b r\rangle)$ are evaluated in standard relational models, and the meaning of all the operators of the basic modal logic is unchanged. When we evaluate formulas containing dynamic operators, we will need to keep track of the edges that have been modified. To that end, let us define precisely the models that we will use. In the rest of this article we will use $w v$ as a shorthand for $\{(w, v)\}$ or $(w, v)$. Context will always disambiguate the intended use.

Definition 2 (Models and Model Updates). $A$ model $\mathcal{M}$ is a triple $\mathcal{M}=$ $\langle W, R, V\rangle$, where $W$ is a non-empty set whose elements are called points or states; $R \subseteq W \times W$ is the accessibility relation; and $V: \mathrm{PROP} \mapsto \mathcal{P}(W)$ is a valuation.

Given a model $\mathcal{M}=\langle W, R, V\rangle$, we define the following notations:

(swapping) $\mathcal{M}_{v w}^{*}=\left\langle W, R_{v w}^{*}, V\right\rangle$, with $R_{v w}^{*}=(R \backslash w v) \cup v w, w v \in R$. (sabotaging) $\mathcal{M}_{w v}^{-}=\left\langle W, R_{w v}^{-}, V\right\rangle$, with $R_{w v}^{-}=R \backslash w v, w v \in R$. (bridging) $\quad \mathcal{M}_{w v}^{+}=\left\langle W, R_{w v}^{+}, V\right\rangle$, with $R_{w v}^{+}=R \cup w v, w v \in(W \times W) \backslash R$.

Let $w$ be a state in $\mathcal{M}$, the pair $(\mathcal{M}, w)$ is called a pointed model; we will usually drop parenthesis and call $\mathcal{M}, w$ a pointed model.

We are now ready to introduce the semantics.

Definition 3 (Semantics). Given a pointed model $\mathcal{M}, w$ and a formula $\varphi$ we say that $\mathcal{M}, w$ satisfies $\varphi$, and write $\mathcal{M}, w \models \varphi$, when

$$
\begin{aligned}
& \mathcal{M}, w \models p \quad \text { iff } w \in V(p) \\
& \mathcal{M}, w \models \neg \varphi \quad \text { iff } \mathcal{M}, w \not \models \varphi \\
& \mathcal{M}, w \models \varphi \wedge \psi \text { iff } \mathcal{M}, w \models \varphi \text { and } \mathcal{M}, w \models \psi \\
& \mathcal{M}, w \models \nabla \varphi \quad \text { iff for some } v \in W \text { s.t. } w R v, \mathcal{M}, v \models \varphi \\
& \mathcal{M}, w \models\langle s w\rangle \varphi \text { iff for some } v \in W \text { s.t. } w R v, \mathcal{M}_{v w}^{*}, v \models \varphi \\
& \mathcal{M}, w \models\langle g s\rangle \varphi \text { iff for some } v, u \in W \text {, s.t. } v R u, \mathcal{M}_{v u}^{-}, w \models \varphi \\
& \mathcal{M}, w \models\langle l s\rangle \varphi \text { iff for some } v \in W \text { s.t. } w R v, \mathcal{M}_{w v}^{-}, v \models \varphi \\
& \mathcal{M}, w \models\langle b r\rangle \varphi \text { iff for some } v \in W \text { s.t. } \neg w R v, \mathcal{M}_{w v}^{+}, v \models \varphi .
\end{aligned}
$$


$\varphi$ is satisfiable if for some pointed model $\mathcal{M}, w$ we have $\mathcal{M}, w \models \varphi$.

We write $\mathcal{M}, w \equiv_{\mathfrak{L}} \mathcal{N}, v$ when both models satisfy the same $\mathfrak{L}$-formulas, i.e., for all $\varphi \in \mathfrak{L}, \mathcal{M}, w \models \varphi$ if and only if $\mathcal{N}, v \models \varphi$. We will drop the $\mathfrak{L}$ subindex when no confusion arises.

Once syntax and semantics are in place, the following result that distinguishes the dynamic logics from $\mathcal{M L}$ can be easily established. A basic result for $\mathcal{M L}$ shows that it has the tree model property: every satisfiable formula of $\mathcal{M L}$ can be satisfied at the root of a model where the accessibility relation defines a tree (i.e., there is a root, the relation is irreflexive, all elements different from the root can be reached from the root via the transitive closure of the accessibility relation, and no element has two different predecessors).

Theorem 4. $\mathcal{M L}(\downarrow)$ does not have the tree model property, for $\in\{\langle s w\rangle,\langle g s\rangle$, $\langle l s\rangle,\langle b r\rangle\}$

Proof. For details see the appendix. We present formulas that ensure that the accessibility relation does not define a tree. The $\langle g s\rangle$ case has already been proved in [6]. Suppose the following formulas hold at some point $w$ :

1. $p \wedge\left(\bigwedge_{1 \leq i<3} \square^{i} \neg p\right) \wedge\langle s w\rangle \diamond \diamond p$,

2. $\diamond \diamond \top \wedge \wedge[l s] \square \perp$,

3. $\diamond \diamond \top \wedge[g s] \square \perp$,

4. $\langle b r\rangle \square \perp$,

then $w$ has a reflexive successor; then $w$ is reflexive; then $w$ is reflexive;

In each case, the formula cannot be satisfied in a tree.

As the four logics we introduced are conservative extensions of $\mathcal{M L}$, the formulas above show that each is strictly more expressive than $\mathcal{M L}$. A natural question is whether these dynamic logics are different from each other. We will use bisimulations to answer this question.

Because we need to keep track of the changes on the accessibility relation that the dynamic operators can introduce, we will define bisimulations as relations that link a point of evaluation together with the current accessibility relation.

Definition 5 (Bisimulations). Given models $\mathcal{M}=\langle W, R, V\rangle$ and $\mathcal{M}^{\prime}=\left\langle W^{\prime}\right.$, $\left.R^{\prime}, V^{\prime}\right\rangle$, together with points $w \in W$ and $w^{\prime} \in W^{\prime}$ we say that they are bisimilar

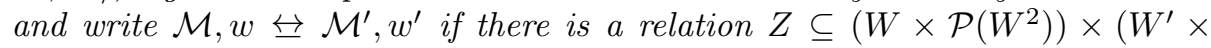
$\left.\mathcal{P}\left(W^{\prime 2}\right)\right)$ such that $(w, R) Z\left(w^{\prime}, R^{\prime}\right)$ satisfying conditions from Figure 1. Which conditions have to be satisfied depends on the operators present in the language.

If needed, we write $\overleftrightarrow{I}_{\mathfrak{L}}$ to indicate that the bisimulation corresponds to $\mathfrak{L}$.

Theorem 6 (Invariance for Dynamic Logics.). For $\mathcal{M L}(\downarrow),\langle\langle s w\rangle,\langle g s\rangle$,

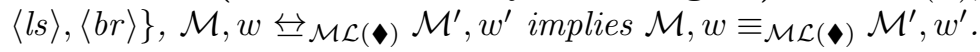

Proof. We will only prove the $\mathcal{M L}(\langle s w\rangle)$ case by structural induction.

The base case holds by (agree), and the $\wedge$ and $\neg$ cases are trivial.

$\left[\nabla \varphi\right.$ case:] Let $\mathcal{M}=\langle W, R, V\rangle$ and $\mathcal{M}^{\prime}=\left\langle W^{\prime}, R^{\prime}, V^{\prime}\right\rangle$. Suppose $\mathcal{M}, w \models \nabla \varphi$. Then there is $v$ in $W$ s.t. $w R v$ and $\mathcal{M}, v \models \varphi$. Since $Z$ is a bisimulation, by 


\begin{tabular}{|c|c|c|}
\hline always & (nontriv) & $Z$ is not empty \\
\hline always & (agree) & $\begin{array}{l}\text { If }(w, S) Z\left(w^{\prime}, S^{\prime}\right), w \text { and } w^{\prime} \text { make the same propositional variables } \\
\text { true. }\end{array}$ \\
\hline \multirow[t]{2}{*}{$\diamond$} & (zig) & If $w S v$, there is $v^{\prime} \in W^{\prime}$ s.t. $w^{\prime} S^{\prime} v^{\prime}$ and $(v, S) Z\left(v^{\prime}, S^{\prime}\right)$ \\
\hline & (zag) & If $w^{\prime} S^{\prime} v^{\prime}$, there is $v \in W$ s.t. $w S v$ and $(v, S) Z\left(v^{\prime}, S^{\prime}\right)$ \\
\hline \multirow[t]{2}{*}{$\langle s w\rangle$} & (sw-zig) & If $w S v$, there is $v^{\prime} \in W^{\prime}$ s.t. $w^{\prime} S^{\prime} v^{\prime}$ and $\left(v, S_{v w}^{*}\right) Z\left(v^{\prime}, S_{v^{\prime} w^{\prime}}^{\prime *}\right)$ \\
\hline & $(s w$-zag) & If $w^{\prime} S^{\prime} v^{\prime}$, there is $v \in W$ s.t. $w S v$ and $\left(v, S_{v w}^{*}\right) Z\left(v^{\prime}, S_{v^{\prime} w^{\prime}}^{* *}\right)$ \\
\hline \multirow[t]{2}{*}{$\langle g s\rangle$} & (gs-zig) & If $v S u$, there is $v^{\prime}, u^{\prime} \in W^{\prime}$ s.t. $v^{\prime} S^{\prime} u^{\prime}$ and $\left(w, S_{v u}^{-}\right) Z\left(w^{\prime}, S_{v^{\prime} u^{\prime}}^{\prime}\right)$ \\
\hline & (gs-zag) & If $v^{\prime} S^{\prime} u^{\prime}$, there is $v, u \in W$ s.t. $v S u$ and $\left(w, S_{v u}^{-}\right) Z\left(w^{\prime}, S_{v^{\prime} u^{\prime}}^{-}\right)$ \\
\hline \multirow[t]{2}{*}{$\langle l s\rangle$} & $(l s$-zig) & If $w S v$, there is $v^{\prime} \in W^{\prime}$ s.t. $w^{\prime} S^{\prime} v^{\prime}$ and $\left(v, S_{w v}^{-}\right) Z\left(v^{\prime}, S_{w^{\prime} v^{\prime}}^{-}\right)$ \\
\hline & $(l s-\mathrm{zag})$ & If $w^{\prime} S^{\prime} v^{\prime}$, there is $v \in W$ s.t. $w S v$ and $\left(v, S_{w v}^{-}\right) Z\left(v^{\prime}, S_{w^{\prime} v^{\prime}}^{-}\right)$ \\
\hline \multirow[t]{2}{*}{$\langle b r\rangle$} & (br-zig) & If $\neg w S v$, there is $v^{\prime} \in W^{\prime}$ s.t. $\neg w^{\prime} S^{\prime} v^{\prime}$ and $\left(v, S_{w v}^{+}\right) Z\left(v^{\prime}, S_{w^{\prime} v^{\prime}}^{\prime+}\right)$ \\
\hline & $(b r$-zag) & If $\neg w^{\prime} S^{\prime} v^{\prime}$, there is $v \in W$ s.t. $\neg w S v$ and $\left(v, S_{w v}^{+}\right) Z\left(v^{\prime}, S_{w^{\prime} v^{\prime}}^{\prime+}\right)$ \\
\hline
\end{tabular}

Fig. 1. Conditions for $\mathcal{M L}(\bullet)$-bisimulations.

(zig) we have $v^{\prime} \in W^{\prime}$ s.t. $w^{\prime} R^{\prime} v^{\prime}$ and $(v, R) Z\left(v^{\prime}, R^{\prime}\right)$. By inductive hypothesis, $\mathcal{M}^{\prime}, v^{\prime} \models \varphi$ and by definition $\mathcal{M}^{\prime}, w^{\prime} \models \Delta \varphi$. For the other direction use (zag). $[\langle s w\rangle \varphi$ case:] For the left to the right direction suppose $\mathcal{M}, w \models\langle s w\rangle \varphi$. Then there is $v \in W$ s.t. $w R v$ and $\mathcal{M}_{v w}^{*}, v \models \varphi$. Because $Z$ is a bisimulation, by $\left(s w\right.$-zig) we have $v^{\prime} \in W^{\prime}$ s.t. $w^{\prime} R^{\prime} v^{\prime}$ and $\left(v, R_{v w}^{*}\right) Z\left(v^{\prime}, R_{v^{\prime} w^{\prime}}^{\prime *}\right)$. By inductive hypothesis, $\mathcal{M}_{v^{\prime} w^{\prime}}^{\prime *}, v^{\prime} \models \varphi$ and by definition $\mathcal{M}^{\prime}, w^{\prime} \models\langle s w\rangle \varphi$. For the other direction use (sw-zag).

\section{Expressive Power}

With the appropriate notions of bisimulation at hand we can now start the comparison of the expressive power of the different dynamic modal logics we introduced. We will use the following standard definition of when a logic is at least as expressive as another.

Definition $7\left(\mathcal{L} \leq \mathcal{L}^{\prime}\right)$. We say that $\mathcal{L}^{\prime}$ is at least as expressive as $\mathcal{L}$ (notation $\left.\mathcal{L} \leq \mathcal{L}^{\prime}\right)$ if there is a function $\operatorname{Tr}$ between formulas of $\mathcal{L}$ and $\mathcal{L}^{\prime}$ such that for every model $\mathcal{M}$ and every formula $\varphi$ of $\mathcal{L}$ we have that

$$
\mathcal{M} \models_{\mathcal{L}} \varphi \text { iff } \mathcal{M} \models_{\mathcal{L}^{\prime}} \operatorname{Tr}(\varphi) .
$$

$\mathcal{M}$ is seen as a model of $\mathcal{L}$ on the left and as a model of $\mathcal{L}^{\prime}$ on the right, and we use in each case the appropriate semantic relation $\models_{\mathcal{L}}$ or $\models_{\mathcal{L}^{\prime}}$ as required.

We say that $\mathcal{L}$ and $\mathcal{L}^{\prime}$ are uncomparable if $\mathcal{L} \not \mathcal{L}^{\prime}$ and $\mathcal{L}^{\prime} \not \leq \mathcal{L}$.

By inspecting suitable models we can establish the following result.

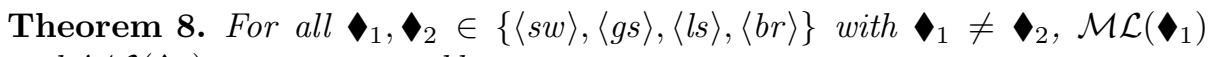
and $\mathcal{M L}\left(\bigotimes_{2}\right)$ are uncomparable. 


\begin{tabular}{|c|c|c|c|}
\hline $\mathcal{M}$ & $\mathcal{M}^{\prime}$ & Distinct by & Bisimilar for \\
\hline$\dot{w}$ & $?$ & $\begin{array}{l}\langle b r\rangle\langle b r\rangle \top \\
\langle g s\rangle \top\end{array}$ & $\begin{array}{l}\mathcal{M L}(\langle l s\rangle) \\
\mathcal{M L} \mathcal{L}(\langle s w\rangle)\end{array}$ \\
\hline ? & & $\begin{array}{l}\langle l s\rangle \diamond \top \\
\langle g s\rangle \diamond \top\end{array}$ & $\begin{array}{l}\mathcal{M L}(\langle s w\rangle) \\
\mathcal{M L}(\langle b r\rangle)\end{array}$ \\
\hline$w$ & $\mathfrak{w}^{\prime}$ & $\begin{array}{l}\langle s w\rangle\langle s w\rangle \diamond \diamond \diamond \square \perp \\
{[b r][b r] \perp}\end{array}$ & $\begin{array}{l}\mathcal{M} \mathcal{L}(\langle g s\rangle) \\
\mathcal{M L}(\langle l s\rangle)\end{array}$ \\
\hline 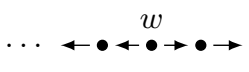 & $w^{\prime} \cdot \longrightarrow \bullet \longrightarrow \bullet \longrightarrow \cdots$ & $\mid\langle s w\rangle \diamond \square \perp$ & $\mathcal{M L}(\langle b r\rangle)$ \\
\hline$\pi$ & & $\langle l s\rangle \diamond \square \perp$ & $\mathcal{M L}(\langle g s\rangle)$ \\
\hline
\end{tabular}

Fig. 2. Bisimilar models and distinguishing formulas.

Proof. In Figure 2 we summarize our results by presenting pairs of models that are bisimilar for a given logic and distinguishable by another. More precisely, the formulas given in the third column are false at $\mathcal{M}, w$ and true at $\mathcal{M}^{\prime}, w^{\prime}$.

That the models are bisimilar for the given logics can be easily verified for the first two rows. In the third row, the given models are bisimilar for $\mathcal{M L}(\langle g s\rangle)$ and $\mathcal{M L}(\langle l s\rangle)$ because they are bisimilar for $\mathcal{M L}$, they are acyclic and (for $\mathcal{M L}(\langle g s\rangle))$ they contain the same number of edges. In the fourth row, both models are $\mathcal{M L}(\langle b r\rangle)$-bisimilar since they are infinite, hence one can add as many links as needed to points that are modally bisimilar.

Finally, the pointed models of the last row are the same graph with a different evaluation point. The graph is a star that has infinitely many ingoing branches, and infinitely many ingoing-outgoing branches. $w$ is a point located at the end of an ingoing branch, and $w^{\prime}$ is at the end of an ingoing-outgoing branch. Let us present the $\mathcal{M L}(\langle g s\rangle)$-bisimulation as a game between Spoiler and Duplicator. If Spoiler moves to the center of the star, Duplicator can do the same and both situations become undistinguishable. If Spoiler deletes one of the ingoing edges that has $w$ or $w^{\prime}$ as origin, then Duplicator does the same on the other graph, and any further edge deletion can also be imitated. If Spoiler deletes the outgoing edge that goes from the center of the graph towards $w^{\prime}$, then Duplicator can delete any outgoing edge without changing the graph, given that there are infinitely many edges of both kinds.

\section{Model Checking Dynamic Logics}

In this section we establish complexity results for the model checking task in the various dynamic modal logics we presented. All the results are established using a 
similar argument: hardness proofs are done by encoding the satisfiability problem of Quantified Boolean Formulas (QBF) [8] as the model checking problem of each logic. While the idea behind the encoding is the same for all the logics involved, the encoding needs to be slightly modified in each case taking into consideration the semantics of the various dynamic operators.

PSpace-hardness for global sabotage was already proved in $[7,6]$, but we provide here a more direct proof.

Theorem 9. For $\in\{\langle s w\rangle,\langle g s\rangle,\langle l s\rangle,\langle b r\rangle\}$, model checking for any of the logics $\mathcal{M L}(\bullet)$ is PSpace-hard.

Proof. We will reduce the PSpace-complete satisfiability problem of Quantified Boolean Formulas (QBF) to the model checking problem of each of these logics. For a complete proof of the case of $\mathcal{M L}(\langle s w\rangle)$, consult the appendix.

Consider $\mathcal{M L}(\langle s w\rangle)$. Let $\alpha$ be a QBF formula with variables $\left\{x_{1}, \ldots, x_{k}\right\}$. Without loss of generality we can assume that $\alpha$ has no free variables and no variable is quantified twice. One can build in polynomial time the relational structure $\mathcal{M}_{k}=\langle W, R, V\rangle$ over a signature with one relational symbol and propositions $\left\{p_{\top}, p_{1}, \ldots, p_{k}\right\}$, where:

$$
\begin{aligned}
& W \quad=\{w\} \cup\left\{w_{i}^{1}, w_{i}^{0} \mid 1 \leq i \leq k\right\} \\
& V\left(p_{i}\right)=\left\{w_{i}^{1}, w_{i}^{0}\right\} \\
& V\left(p_{\top}\right)=\left\{w_{i}^{1} \mid 1 \leq i \leq k\right\} \\
& R \quad=\left\{\left(w, w_{i}^{1}\right),\left(w, w_{i}^{0}\right) \mid 1 \leq i \leq k\right\}
\end{aligned}
$$

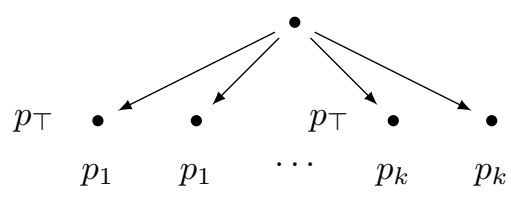

Let ()$^{\prime}$ be the following linear translation from $\mathrm{QBF}$ to $\mathcal{M L}(\langle s w\rangle)$

$$
\begin{array}{ll}
\left(\exists x_{i} \cdot \alpha\right)^{\prime} & =\langle s w\rangle\left(p_{i} \wedge \diamond(\alpha)^{\prime}\right) \\
\left(x_{i}\right)^{\prime} & =\neg \nabla\left(p_{i} \wedge p \top\right) \\
(\neg \alpha)^{\prime} & =\neg(\alpha)^{\prime} \\
(\alpha \wedge \beta) & =(\alpha)^{\prime} \wedge(\beta)^{\prime} .
\end{array}
$$

It remains to see that $\alpha$ is satisfiable if, and only if, $\mathcal{M}_{k}, w \models(\alpha)^{\prime}$ holds. This part of the proof is in the appendix. This shows that the model checking problem of $\mathcal{M L}(\langle s w\rangle)$ is PSpace-hard.

For $\mathcal{M L}(\langle g s\rangle)$ and $\mathcal{M L}(\langle l s\rangle)$, we use the following model:

$$
\begin{aligned}
& W \quad=\{w\} \cup\left\{w_{i}^{1}, w_{i}^{0} \mid 1 \leq i \leq k\right\} \\
& V\left(p_{i}\right)=\left\{w_{i}^{1}, w_{i}^{0}\right\} \\
& V\left(p_{\top}\right)=\left\{w_{i}^{1} \mid 1 \leq i \leq k\right\} \\
& R=\left\{\left(w, w_{i}^{1}\right),\left(w, w_{i}^{0}\right),\left(w_{i}^{1}, w\right),\left(w_{i}^{0}, w\right) \quad p_{1} \quad p_{1} \quad \cdots \quad p_{k} \quad p_{k}\right. \\
& \mid 1 \leq i \leq k\}
\end{aligned}
$$

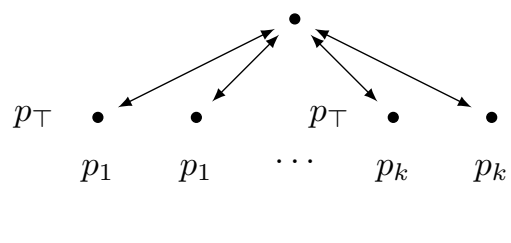

Let ()$^{\prime}$ be the following linear translation from $\mathrm{QBF}$ to $\mathcal{M L}(\langle l s\rangle)$ :

$$
\begin{aligned}
& \left(\exists x_{i} . \alpha\right)^{\prime}=\langle l s\rangle\left(p_{i} \wedge \diamond(\alpha)^{\prime}\right) \\
& \left(x_{i}\right)^{\prime}=\neg \diamond\left(p_{i} \wedge p_{\top}\right) \\
& (\neg \alpha)^{\prime}=\neg(\alpha)^{\prime} \\
& (\alpha \wedge \beta)=(\alpha)^{\prime} \wedge(\beta)^{\prime} .
\end{aligned}
$$


From $\mathrm{QBF}$ to $\mathcal{M L}(\langle g s\rangle)$, we provide the following translation:

$$
\begin{aligned}
& \left(\exists x_{i} . \alpha\right)^{\prime}=\langle g s\rangle\left(\left(\neg \nabla\left(p_{i} \wedge p_{\top}\right) \vee \neg \nabla\left(p_{i} \wedge \neg p_{\top}\right)\right) \wedge \diamond\left(p_{i} \wedge \diamond(\alpha)^{\prime}\right)\right) \\
& \left(x_{i}\right)^{\prime}=\neg \nabla\left(p_{i} \wedge p_{\top}\right) \\
& (\neg \alpha)^{\prime}=\neg(\alpha)^{\prime} \\
& (\alpha \wedge \beta)=(\alpha)^{\prime} \wedge(\beta)^{\prime} .
\end{aligned}
$$

In both cases, showing that a QBF formula $\alpha$ is satisfiable if, and only if, $\mathcal{M}_{k}, w \models(\alpha)^{\prime}$ holds can be done similarly to the case of $\mathcal{M L}(\langle s w\rangle)$.

Finally, to prove PSpace-hardness for $\mathcal{M L}(\langle b r\rangle)$, build the following model:

$$
\begin{aligned}
& W=\{w\} \cup\left\{w_{i}^{1}, w_{i}^{0} \mid 1 \leq i \leq k\right\} \\
& V\left(p_{i}\right)=\left\{w_{i}^{1}, w_{i}^{0}\right\} \\
& V\left(p_{\top}\right)=\left\{w_{i}^{1} \mid 1 \leq i \leq k\right\} \\
& R \quad=\left\{\left(w_{i}^{1}, w\right),\left(w_{i}^{0}, w\right) \mid 1 \leq i \leq k\right\}
\end{aligned}
$$

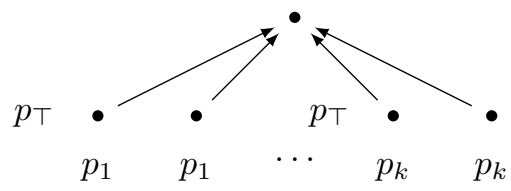

And use the following linear translation ( )':

$$
\begin{array}{ll}
\left(\exists x_{i} \cdot \alpha\right)^{\prime} & =\langle b r\rangle\left(p_{i} \wedge \diamond(\alpha)^{\prime}\right) \\
\left(x_{i}\right)^{\prime} & =\diamond\left(p_{i} \wedge p_{\top}\right) \\
(\neg \alpha)^{\prime} & =\neg(\alpha)^{\prime} \\
(\alpha \wedge \beta) & =(\alpha)^{\prime} \wedge(\beta)^{\prime} .
\end{array}
$$

Theorem 10. Model checking for $\mathcal{M L}(\langle s w\rangle,\langle g s\rangle,\langle l s\rangle,\langle b r\rangle)$ is in PSpace.

Proof. The evaluation of the truth of a formula in a model can be done by a polynomial space algorithm that follows Definition 3.

The algorithm works on the same copy of the model, except when dealing with formulas whose main connector is $\langle s w\rangle,\langle g s\rangle,\langle l s\rangle$ or $\langle b r\rangle$ (i.e., dynamic operators). In such cases, by proceeding depth-first among at most $|W|$ possible choices, the algorithm only allocates as much additional space as the size of the initial model to store the modified copy. This memory can be reclaimed once the result of the recursive call is known. The maximum number of copies of the input model in memory is bounded by the nesting of dynamic operators of the input formula. Hence the algorithm runs using only polynomial space.

With the previous results we get:

Theorem 11. For $\in\{\langle s w\rangle,\langle g s\rangle,\langle l s\rangle,\langle b r\rangle\}$, model checking for any of the logics $\mathcal{M L}(\diamond)$ is PSpace-complete.

\section{Conclusions}

In this article we investigate dynamic modal logics that can modify the model during the evaluation of a formula. Dynamic Epistemic Logics (DEL) as those investigated in $[9,5,11,13]$ are well known examples of languages which can also update the model during evaluation. The standard update operation used in DELs is to move evaluation to a submodel defined by a certain 'announcement', 
e.g., to the model representing the fact that $\varphi$ is now known, obtained as the restriction to all the nodes satisfying a formula $\varphi$. Instead, in this article we investigate logics that can explicitly modify the accessibility relation, as the sabotage logics first introduced by van Benthem in [12].

We introduce a number of operators with both local and global effects, and which can add, delete and modify edges in the accessibility relation. The goal was to investigate the different degrees of liberty that the operators offered, and how much overlap there was between the logics they defined, and the models they could describe.

We show in Sections 2 and 3 that the languages obtained by the extension of the basic modal logic with each of the dynamic operators can be characterized using bisimulations. Actually, even though each operator requires a particular pair of zig and zag conditions, the definition is modular and the set up homogeneous. All the bisimulations involved are of the same type, linking a pair of point of evaluation and accessibility relation in one model, with a similar pair in the other. Moreover, a suitable definition of bisimulation for the basic modal logic extended with any combination of the new dynamic operators can be obtained by using the adequate zig and zag conditions associated to the operators involved. Summing up then, even though the logics obtained are different in each case, they are all amenable to fairly classical modal analysis.

In Section 4 we turn to model checking, and show that the complexity of this reasoning task is PSpace-complete for all the logics considered. Once more, the proofs are fairly homogeneous in all cases. The general set up is the encoding of the PSpace-complete QBF satisfiability problem in each of the logics. In each case, a suitable representation for the assignment and the concrete translation used needs to be defined, but once this is done the proof is similar.

More precisely, we established the complexity of the combined model checking task, measured in function of the length of an input model and an input formula. It is also possible to consider the task of model checking against a fixed model, measuring its complexity in function of the size of an input formula (this is known as the formula complexity). One can also fix a formula and measure the complexity of model checking in function of the length of an input model (known as the program complexity or data complexity). Both notions were introduced in [14], and it has been shown in [6] that the formula complexity and the program complexity of $\mathcal{M L}(\langle g s\rangle)$ are respectively linear and polynomial. We believe that the proof generalizes to $\mathcal{M L}(\langle l s\rangle), \mathcal{M L}(\langle s w\rangle)$ and $\mathcal{M L}(\langle b r\rangle)$ with identical results.

Another natural direction for future research would be to investigate the complexity of the satisfiability problem of these logics. From [6], we already know that $\mathcal{M L}(\langle g s\rangle)$ is undecidable. We conjecture that using techniques from $[3,1]$, it is possible to prove that the problem is undecidable in all remaining cases.

Acknowledgments: This work was partially supported by grants ANPCyTPICT-2008-306, ANPCyT-PIC-2010-688, the FP7-PEOPLE-2011-IRSES Project 
"Mobility between Europe and Argentina applying Logics to Systems" (MEALS) and the Laboratoire Internationale Associé "INFINIS".

\section{References}

1. Areces, C., Figueira, D., Figueira, S., Mera, S.: The expressive power of memory logics. Review of Symbolic Logic 4(2), 290-318 (2011)

2. Blackburn, P., de Rijke, M., Venema, Y.: Modal Logic. Cambridge University Press (2001)

3. Blackburn, P., Seligman, J.: Hybrid languages. Journal of Logic, Language and Information 4, 251-272 (1995)

4. Blackburn, P., Wolter, F., van Benthem, J. (eds.): Handbook of Modal Logics. Elsevier (2006)

5. Gerbrandy, J.: Bisimulations on Planet Kripke. Ph.D. thesis, University of Amsterdam (1999), ILLC Dissertation series DS-1999-01

6. Löding, C., Rohde, P.: Model checking and satisfiability for sabotage modal logic. In: Pandya, P., Radhakrishnan, J. (eds.) FSTTCS. Lecture Notes in Computer Science, vol. 2914, pp. 302-313. Springer (2003)

7. Löding, C., Rohde, P.: Solving the sabotage game is PSPACE-hard. In: Mathematical Foundations of Computer Science 2003, Lecture Notes in Computer Science, vol. 2747, pp. 531-540. Springer, Berlin (2003)

8. Papadimitriou, C.: Computational Complexity. Addison-Wesley (1994)

9. Plaza, J.: Logics of public communications. Synthese 158(2), 165-179 (2007)

10. Rohde, P.: On games and logics over dynamically changing structures. Ph.D. thesis, RWTH Aachen (2006)

11. van Benthem, J.: Logics for information update. In: TARK'01: Proceedings of the 8th Conference on Theoretical Aspects of Rationality and Knowledge. pp. 51-67. Morgan Kaufmann Publishers Inc. (2001)

12. van Benthem, J.: An essay on sabotage and obstruction. In: Mechanizing Mathematical Reasoning. pp. 268-276 (2005)

13. van Ditmarsch, H., van der Hoek, W., Kooi, B.: Dynamic Epistemic Logic. Kluwer (2007)

14. Vardi, M.Y.: The complexity of relational query languages (extended abstract). In: Lewis, H.R., Simons, B.B., Burkhard, W.A., Landweber, L.H. (eds.) STOC. pp. 137-146. ACM (1982)

\section{Appendix}

Theorem 4. $\mathcal{M L}(\diamond)$ does not have the tree model property, for $\diamond \in\{\langle s w\rangle,\langle g s\rangle$, $\langle l s\rangle,\langle b r\rangle\}$

Proof. We are going to list formulas that force no-treelike models:

1. The formula

$$
\varphi=p \wedge\left(\bigwedge_{1 \leq i \leq 3} \square^{i} \neg p\right) \wedge\langle s w\rangle \diamond \diamond p
$$

is true at a state $w$ in a model, only if $w$ has a reflexive successor. 
Suppose we evaluate $\varphi$ at some state $w$ of an arbitrary model. The 'static' part of the formula $p \wedge\left(\bigwedge_{1<i<3} \square^{i} \neg p\right)$ makes sure that $p$ is true in $w$ and that no $p$ state is reachable within three steps from $w$ (in particular, $w$ cannot be reflexive).

Because $\langle s w\rangle \diamond \diamond p$ is true at $w$, there should be an $R$-successor $v$ where $\diamond \diamond p$ holds once the accessibility relation has been updated to $R_{v w}^{*}$. That is, $v$ has to reach a $p$-state in exactly two $R_{v w}^{*}$-steps. But the only $p$-state sufficiently close is $w$ which is reachable in one step. As $w$ is not reflexive, $v$ has to be reflexive so that we can linger at $v$ for one loop and reach $p$ in the correct number of states.

2. The formula

$$
\varphi=\diamond \diamond \top \wedge[l s] \square \perp
$$

is true at a state $w$ in a model, only if $w$ is reflexive.

Suppose we evaluate $\varphi$ at some state $w$ of an arbitrary model. On one hand, the 'static' part of the formula $\diamond \diamond T$ ensures it is possible to take two accessibility relations. On the other hand, the 'dynamic' part of the formula $[l s] \square \perp$ tells us that after taking any accessibility relation and eliminating it, it is no longer possible to go anywhere else. This can only happen if the point $w$ is reflexive and does not have any other outgoing links.

3. The formula

$$
\varphi=\diamond \diamond \top \wedge[g s] \square \perp
$$

(from [6]) is true at a state $w$ in a model, only if $w$ is reflexive.

4. The formula

$$
\varphi=\langle b r\rangle \square \perp
$$

is only satisfiable in models that have at least two unconnected points.

Theorem 11. Model checking for $\mathcal{M L}(\langle s w\rangle)$ is PSpace-hard.

Proof. We will reduce the PSpace-complete satisfiability problem of Quantified Boolean Formulas $(\mathrm{QBF})$ to the model checking problem of $\mathcal{M L}(\langle s w\rangle)$.

Let $\alpha$ be a $\mathrm{QBF}$ formula with variables $\left\{x_{1}, \ldots, x_{k}\right\}$. Without loss of generality we can assume that $\alpha$ has no free variables and no variable is quantified twice. One can build in polynomial time the relational structure $\mathcal{M}_{k}=\langle W, R, V\rangle$ over a signature with one relational symbol and propositions $\left\{p_{\top}, p_{1}, \ldots, p_{k}\right\}$, where:

$$
\begin{aligned}
& W \\
& W\left(p_{i}\right)=\left\{w_{i}^{1}, w_{i}^{0}\right\} \\
& V\left(p_{\top}\right)=\left\{w_{i}^{1} \mid 1 \leq i \leq k\right\} \\
& R \quad=\left\{\left(w, w_{i}^{1}\right),\left(w, w_{i}^{0}\right) \mid 1 \leq i \leq k\right\}
\end{aligned}
$$

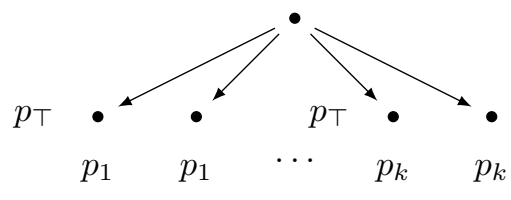

Let ( $)^{\prime}$ be the following linear translation from $\mathrm{QBF}$ to $\mathcal{M L}(\langle s w\rangle)$

$$
\begin{aligned}
& \left(\exists x_{i} . \alpha\right)^{\prime}=\langle s w\rangle\left(p_{i} \wedge \diamond(\alpha)^{\prime}\right) \\
& \left(x_{i}\right)^{\prime}=\neg \diamond\left(p_{i} \wedge p_{\top}\right) \\
& (\neg \alpha)^{\prime}=\neg(\alpha)^{\prime} \\
& (\alpha \wedge \beta)=(\alpha)^{\prime} \wedge(\beta)^{\prime} .
\end{aligned}
$$


It remains to see that $\alpha$ is satisfiable iff $\mathcal{M}_{k}, w \models(\alpha)^{\prime}$ holds. Let us write $v \models_{\text {qbf }} \alpha$ if valuation $v:\left\{x_{1}, \ldots, x_{k}\right\} \rightarrow\{0,1\}$ satisfies $\alpha$. For a model $\mathcal{M}$ with relation $R$ we define $v_{R}:\left\{x_{1}, \ldots, x_{k}\right\}$ as " $v_{R}\left(x_{i}\right)=1$ iff $\left(w, w_{i}^{1}\right) \notin R$ ", in the present case, iff the link between $w$ and $w_{i}^{1}$ has been swapped.

Let $\beta$ be any subformula of $\alpha$. We will show by induction on $\beta$ that $\mathcal{M}, w \models$ $(\beta)^{\prime}$ iff $v_{R} \models_{\mathrm{qbf}} \beta$. The first observation is that $R$ satisfies i) if $x_{i}$ is free in $\beta$, then $\left(w, w_{i}^{1}\right) \notin R$ or $\left(w, w_{i}^{0}\right) \notin R$ but not both, and ii) if $x_{i}$ is not free in $\beta$ then $\left(w, w_{i}^{1}\right) \in R$ and $\left(w, w_{i}^{0}\right) \in R$. From here it will follow that $\mathcal{M}_{k}, w \models(\alpha)^{\prime}$ iff $v \models_{\text {qbf }} \alpha$ for any $v$ since $\alpha$ has no free variables, iff $\alpha$ is satisfiable.

For the base case, $v_{R} \models_{\mathrm{qbf}} x_{i}$ iff $\left(w, w_{i}^{1}\right) \notin R$ which implies (from the definition of $\left.\mathcal{M}_{k}\right) \mathcal{M}, w \models\left(x_{i}\right)^{\prime}$. For the other direction, suppose $\mathcal{M}, w \not \models\left(x_{i}\right)^{\prime}$. Hence $\mathcal{M}, w \models \diamond\left(p_{i} \wedge p_{\top}\right)$ which implies $\left(w, w_{i}^{1}\right) \in R$ and $u_{R} \nvdash_{\text {qbf }} x_{i}$.

The boolean cases follow directly from the inductive hypothesis.

Consider the case $\beta=\exists x_{i} \cdot \gamma$. Since no variable is bound twice in $\alpha$ we know $\left(w, w_{x_{i}}^{1}\right) \in R$ and $\left(w, w_{i}^{0}\right) \in R$. We have $v_{R} \models_{\mathrm{qbf}} \beta$ iff $\left(v_{R}\left[x_{i} \mapsto 0\right] \models_{\mathrm{qbf}} \gamma\right.$ or $\left.v_{R}\left[x_{i} \mapsto 1\right] \models_{\mathrm{qbf}} \gamma\right)$ iff $\left(v_{R^{w_{i}^{0} w}} \models_{\mathrm{qbf}} \gamma\right.$ or $\left.v_{R^{w_{i}^{1} w}} \models_{\mathrm{qbf}} \gamma\right)$. By inductive hypothesis, this is the case if and only if $\left(\mathcal{M}^{w_{i}^{0} w}, w_{i}^{0} \models \diamond(\gamma)^{\prime}\right.$ or $\left.\mathcal{M}^{w_{i}^{1} w}, w_{i}^{1} \models \diamond(\gamma)^{\prime}\right)$ iff $\mathcal{M}, w \models\langle s w\rangle\left(p_{i} \wedge \diamond(\gamma)^{\prime}\right)$ iff $\mathcal{M}, w \models\left(\exists x_{i} \cdot \gamma\right)^{\prime}$. 